\title{
Desempenho intelectual na exposição alcoólica fetal: relato de série de 10 casos
}

\author{
Intellectual performance in fetal alcohol exposure: report of 10 cases series
}

Vanessa Karam de Lima Ferreira', Gisele Viegas Dias Ferreira', José Mauro Bras de Lima², Marcelo Santos Cruz'

\section{RESUMO}

Objetivo: Descrever o desempenho intelectual em uma amostra de crianças e adolescentes que sofreram exposição pré-natal ao álcool. Métodos: Este estudo se constitui em uma série de casos, composta por 10 sujeitos entre 6 e 16 anos, com histórico de exposição alcoólica fetal. Esses sujeitos foram submetidos a uma avaliação médica para serem caracterizados como amostra clínica, respeitando critérios de inclusão e exclusão previamente estabelecidos. 0 desempenho intelectual da amostra foi estudado por meio dos instrumentos: Escala de Inteligência Wechsler para Criança (WISC-III) e Teste Matrizes Progressivas de Raven. Resultados: A amostra foi caracterizada com desempenho médio inferior pela WISC-III. No Teste Raven, a amostra apresentou desempenho intelectualmente médio. Entre todos os índices da WISC-III, Resistência à Distração apresentou-se mais prejudicado. Os subtestes Aritmética e Arranjo de Figuras apresentaram resultados mais prejudicados comparados ao desempenho obtido nos outros subtestes. O prejuízo dessas funções pode trazer como consequência secundária e dificuldades no aprendizado e na interação social e impactar o desenvolvimento desses jovens. Conclusões: Os resultados encontrados nesta série de 10 casos foram comparados àqueles descritos em estudos nacionais e internacionais. O prejuízo cognitivo causado pelo uso do álcool na gestação pode se manifestar em crianças em idade escolar por meio da dificuldade de aprendizagem e da dificuldade de estabelecer interações sociais. Dessa forma, a exposição pré-natal ao álcool torna-se um problema de saúde pública, que precisa de maior atenção das políticas públicas nacionais para a realização de identificação e intervenção precoce.

\section{ABSTRACT}

Objective: Describe the intellectual performance in a sample of children and adolescents that suffered prenatal exposure of alcohol. Methods: This report constitutes a case series study, comprising 10 subjects between 6 and 16 years old, with a history of fetal alcohol exposure. These subjects underwent a medical evaluation to be characterized as a clinical sample, respecting the inclusion and exclusion criteria previously established. The sample's intellectual performance was studied by means of instruments: Wechsler Intelligence Scale for Children 3 ${ }^{\text {rd }}$. Edition (WISC-III) and Raven's Progressive Matrices Test. Results: The sample was evaluated as being of low medium performance by the WISC-III. In the Raven Test the sample exhibited medium intellectual performance. Among all of the WISC-III indexes,

1 Universidade Federal do Rio de Janeiro (UFRJ), Instituto de Psiquiatria, Programa de Estudos e Assistência ao Uso Indevido de Drogas. 2 UFRJ, Hospital Escola São Francisco de Assis. 


\section{Keywords}

Intellectual performance, fetal alcohol syndrome, fetal alcohol spectrum disorders, neuropsychology, fetal alcohol exposure.
Resistance to Distractions was most impaired. The Arithmetic and Picture Arrangement subtests showed more impairment results in comparison of the others subtests. As a secondary consequence, the impairment of these functions can cause difficulties in learning and social interaction and impact the development of these young people. Conclusions: The results of these series of ten cases were compared to those reported in national and international studies. The cognitive impairment caused by alcohol use during pregnancy can manifest in school-age children through learning difficulties and difficulty in establishing social interactions. Thus, prenatal exposure to alcohol becomes a public health issue which needs greater attention from national policies to carry out identification and early intervention.

\section{INTRODUÇÃO}

A síndrome alcoólica fetal (SAF) é consequência da ação teratológica tóxico-metabólica do álcool sobre o embrião, secundária à ingestão de bebida alcoólica pela mãe durante a gravidez' .

A exposição pré-natal ao álcool pode causar os seguintes prejuízos: atraso no desenvolvimento (deficiência de crescimento pré e pós-natal, baixo peso em relação à altura), anomalias faciais (fissura palpebral curta, lábio superior fino, face plana) e prejuízos no sistema nervoso central (microcefalia, prejuízo intelectual) $)^{2}$.

A SAF é caracterizada e diagnosticada pela presença de deficiência no crescimento, características faciais típicas e sinais de disfunções no sistema nervoso central. Crianças que apresentam algumas dessas características, mas não fecham o diagnóstico, são descritas como apresentando efeitos alcoólicos fetais (fetal alcohol effects - FAE). O transtorno do neurodesenvolvimento relacionado ao álcool inclui aqueles que têm alterações no sistema nervoso central, sem apresentar deficiência no crescimento nem características faciais. O termo "transtorno do espectro alcoólico fetal" agrupa todas essas classificações, representando um continuum, no qual a SAF é a forma mais grave ${ }^{3,4}$.

Segundo a Organização Mundial da Saúde ${ }^{5}$, apesar de ainda não existir uma estimativa de prevalência mundial confiável da SAF, um estudo realizado nos Estados Unidos em 2005 aponta para uma prevalência global de 0,97 por 1.000 nascidos vivos. No Brasil, estima-se que nasçam, anualmente, cerca de 3.000 a 9.000 crianças com SAF6.

Os custos sociais consequentes da exposição alcoólica fetal envolvem assistência médica, psicológica, jurídica, além de prejuízo no desempenho escolar, entre outras. Estima-se que em 1998, nos Estados Unidos, as despesas totalizaram US\$ 4,0 bilhões/ano ${ }^{7}$.

Em diversas pesquisas, o desempenho intelectual mostrou-se prejudicado na população exposta ao álcool durante a gestação $0^{8-10}$. Apesar disso, dois estudos encontraram desempenho intelectual preservado, ou seja, quociente de inteligência (QI) de acordo com o esperado para a faixa etária ${ }^{11-12}$.

No Brasil, são escassas as pesquisas sobre o tema. Elas se fazem necessárias, uma vez que o Brasil é um dos países de maior consumo e produção de bebida alcoólica. O consumo de álcool está se dando mais precocemente e tem aumentando na população feminina ${ }^{13}$.

Em relação às políticas públicas brasileiras, ainda não surgiu nenhuma que mencione os riscos que o consumo de álcool pode apresentar para o feto, nem mesmo no recente Decreto-lei no 6.117/2007, que aprovou a Política Nacional sobre o Álcool ${ }^{6}$.

A falta de políticas públicas pode estar impondo barreiras para a identificação e a prevenção da SAF no Brasil, uma vez que ainda há pouco conhecimento sobre os prejuízos causados pela exposição alcoólica fetal, tanto pela população geral, quanto pelos profissionais da saúde.

O objetivo deste artigo é apresentar os resultados da avaliação do desempenho intelectual em uma amostra de crianças/adolescentes que sofreram exposição alcoólica fetal.

\section{MÉTODOS}

Este estudo descreve o desempenho intelectual de uma série de 10 casos.

Participaram do estudo sujeitos de 6 a 16 anos, com histórico de exposição alcoólica fetal. Selecionaram-se crianças e adolescentes que foram encaminhadas para atendimento ambulatorial no Centro de Ensino, Pesquisa e Referência de Alcoologia e Adictologia (Cepral) do Hospital Escola da Universidade Federal do Rio de Janeiro (HESFA/UFRJ).

Na tabela 1, foram relacionados os critérios de inclusão e exclusão da amostra.

Tabela 1. Critérios de inclusão e exclusão para compor amostra clínica

\begin{tabular}{|c|c|}
\hline Critérios de inclusão & Critérios de exclusão \\
\hline $\begin{array}{l}\text { 1. Sujeitos, cujas } \\
\text { mães ingeriram } \\
\text { bebida alcoólica } \\
\text { durante a gestação } \\
\text { 2. Sujeitos } \\
\text { que estejam } \\
\text { devidamente } \\
\text { matriculados e } \\
\text { frequentando a } \\
\text { escola }\end{array}$ & $\begin{array}{l}\text { 1. Sujeitos, cujas mães: } \\
\text { a) consumiram outro tipo de droga, exceto álcool e tabaco, } \\
\text { durante a gravidez } \\
\text { b) relatem história de qualquer tipo de doença que afete } 0 \\
\text { desempenho cognitivo da criança } \\
\text { 2. Sujeitos: } \\
\text { a) que não conseguem realizar as tarefas propostas } \\
\text { b) que possuem histórico de doenças genéticas na família, } \\
\text { para diagnóstico diferencial: síndrome de Cornélia de Lange, } \\
\text { síndrome de Noonan }{ }^{14} \text {, síndrome de Willians, síndrome de } \\
\text { Aarskog, síndrome de Dubowitz, síndrome de Bloom, síndrome } \\
\text { de Turner, síndrome de 0pitz }{ }^{15} \text {, síndrome de velocardiofacial, } \\
\text { síndrome de blepharophimosis e síndrome de Dubowitz }\end{array}$ \\
\hline
\end{tabular}


Os sujeitos que compuseram essa amostra foram diagnosticados com síndrome alcoólica fetal por meio do exame médico, por um psiquiatra treinado, atendendo também aos critérios de inclusão e exclusão descritos na tabela 1. O diagnóstico foi baseado pela observação de todas as características clínicas presentes na SAF: deficiência no crescimento, características faciais típicas e sinais de disfunções no sistema nervoso central, além do relato de uso de álcool materno durante a gestação.

Após a seleção dos sujeitos, foram aplicados os seguintes instrumentos: entrevista semiestruturada, Escala de Inteligência Wechsler para Crianças - terceira edição (WISC-III) e Matrizes Progressivas de Raven (forma geral e especial).

A WISC-III e o teste Matrizes Progressivas de Raven foram escolhidos por serem de ampla utilização em pesquisas que avaliam as funções cognitivas superiores e por terem vários estudos que apontam correlações positivas e significativas entre os dois instrumentos na avaliação do Q17,18.

\section{- WISC-III (Escala de Inteligência Wechsler para Crianças - terceira edição)}

A WISC-III avalia a capacidade cognitiva global de sujeitos entre 6 anos e 16 anos e 11 meses. A bateria é composta de 13 subtestes, cada um avaliando uma dimensão específica da cognição. O desempenho nesses vários subtestes é resumido em três medidas: QI Verbal (QIV), QI de Execução (QIE) e QI Total (QIT), que oferecem estimativas das capacidades cognitivas do indivíduo ${ }^{17}$.

A WISC-III oferece também índices adicionais que avaliam: Compreensão Verbal (CV), Organização Perceptual $(\mathrm{OP})$, Resistência à Distração (RD) e Velocidade de Processamento $(\mathrm{VP})^{17}$.

\section{- Matrizes Progressivas de Raven}

As Matrizes Progressivas de Raven constituem-se em um teste de inteligência não verbal, que avalia o raciocínio analógico, isto é, a habilidade de deduzir relações entre objetos ou elementos. Podem ser empregadas em pessoas de diferentes idades, níveis de instrução, contextos socioculturais e condições físicas ${ }^{18}$.

Esse projeto foi submetido e aceito pelo Comitê de Ética em Pesquisa do Instituto de Psiquiatria da Universidade Federal do Rio de Janeiro (código: 27LIV3-10). Todos os responsáveis assinaram o termo de consentimento livre e esclarecido, autorizando a participação dos sujeitos, e os próprios participantes, as crianças e adolescentes entre 6 e 16 anos, assinaram o termo de assentimento.

\section{RESULTADOS}

Nas tabelas 2 e 3, estão expostos a caracterização da amostra e o consumo de álcool materno durante a gestação, respectivamente.

A tabela 4 mostra o desempenho cognitivo dos sujeitos avaliados. Na WISC-III, de forma geral, os sujeitos obtiveram desempenho limítrofe (QIT = 70 a 79), avaliado pela média do QIT. Quatro sujeitos se enquadraram na classificação de deficiência intelectual (QIT $=\leq 69)$. Os outros seis sujeitos apresentaram desempenhos que variaram desde a faixa limítrofe até a média (QIT = 90 a 109).

No teste Matrizes Progressivas de Raven, de forma geral, os sujeitos apresentaram desempenho dentro da faixa "intelectualmente médio" (percentil = 26 a 74). Três sujeitos apresentaram seus resultados dentro da faixa "intelectualmente deficiente" (percentil $=\leq 5$ ). Dessas três crianças, duas delas já haviam apresentado resultados dentro da faixa de deficiência intelectual na WISC-III (Tabela 4).

Em relação aos índices fatoriais da WISC-III, a RD obteve a menor média (M: 72,8; DP: 8,17), sendo classificada dentro da faixa limítrofe $(Q \mathrm{Q}=70$ a 79$)$. Isso demonstra uma dificuldade na memória auditivo-verbal e imediata, como também no processamento sequencial, com alta correlação com a habilidade matemática.

Tabela 2. Caracterização da amostra exposta ao álcool durante a gestação

\begin{tabular}{|c|c|c|c|c|c|c|c|}
\hline & Sexo & Idade & Escolaridade & Escola & Cuidadora & Medicação & Classe Social \\
\hline Paciente A & M & 10 & $4^{\circ}$ ano & Pública & Mãe biológica & Risperidona & D \\
\hline Paciente B & M & 9 & $4^{\circ}$ ano & Pública & Familiar & Metilfenidato & $C 2$ \\
\hline Paciente C & M & 8 & $1^{\circ}$ ano & Particular & Familiar & Imipramina & $C 2$ \\
\hline Paciente D & $\mathrm{F}$ & 16 & $8^{\circ}$ ano & Pública & Vizinha & $\begin{array}{l}\text { Carbamazepina e } \\
\text { Levomepromazina }\end{array}$ & D \\
\hline Paciente E & M & 8 & $3^{\circ}$ ano & Pública & Mãe biológica & Risperidona & D \\
\hline Paciente F & M & 12 & $6^{\circ}$ ano & Particular & Mãe biológica & Não & B2 \\
\hline Paciente G & M & 6 & $1^{\circ}$ ano & Pública & Mãe biológica & Não & D \\
\hline Paciente $\mathrm{H}$ & $\mathrm{F}$ & 6 & $1^{\circ}$ ano & Pública & Mãe biológica & Não & C2 \\
\hline Paciente I & M & 11 & $5^{\circ}$ ano & Pública & Familiar & Não & C2 \\
\hline Paciente J & $\mathrm{F}$ & 8 & $2^{\circ}$ ano & Pública & Mãe biológica & Não & D \\
\hline
\end{tabular}

M: masculino; F: feminino. 
Tabela 3. Caracterização do consumo de álcool materno durante o período gestacional

\begin{tabular}{|c|c|c|c|c|c|}
\hline & Consumo & Período & Bebida & Frequência & Doses por episódio \\
\hline Paciente A & Álcool & Toda a gravidez & Cerveja & $2 \times$ por semana & 5 ou mais \\
\hline Paciente B & Álcool e tabaco & Até $2^{\circ}$ trimestre & Vodca & NS & NS \\
\hline Paciente C & Álcool e tabaco & NS & NS & NS & NS \\
\hline Paciente D & Álcool & Toda a gravidez & Várias & Todos os dias & 5 ou mais \\
\hline Paciente E & Álcool & Até $3^{\circ}$ trimestre & Cerveja & $1 \mathrm{x}$ por semana & 5 ou mais \\
\hline Paciente F & Álcool e tabaco & Toda a gravidez & Cerveja & $1 \times$ por semana & 5 ou mais \\
\hline Paciente G & Álcool e tabaco & Toda a gravidez & Cerveja & 1 a 2 x por mês & NS \\
\hline Paciente $\mathrm{H}$ & Álcool e tabaco & Toda a gravidez & Cerveja, vinho, caipirinha & $2 \times$ por mês & 4 doses \\
\hline Paciente I & Álcool e tabaco & NS & NS & NS & NS \\
\hline Paciente J & Álcool e tabaco & Até $2^{\circ}$ trimestre & Cerveja & $1 \times$ por semana & 5 ou mais \\
\hline
\end{tabular}

Tabela 4. Tabela de desempenho do WISC-III e Raven

\begin{tabular}{|c|c|c|c|c|c|}
\hline & \multicolumn{4}{|c|}{ WISC III } & \multirow{2}{*}{$\begin{array}{l}\text { Raven } \\
\text { Percentil }\end{array}$} \\
\hline & QIV & QIE & QIT & Percentil & \\
\hline Paciente A & 66 & 60 & 60 & 0,4 & 30 \\
\hline Paciente B & 71 & 63 & 64 & 1 & 30 \\
\hline Paciente C & 71 & 51 & 58 & 0,3 & 5 \\
\hline Paciente D & 65 & 53 & 56 & 0,2 & 1 \\
\hline Paciente E & 99 & 105 & 102 & 55 & 90 \\
\hline Paciente F & 89 & 97 & 92 & 30 & 5 \\
\hline Paciente G & 83 & 82 & 81 & 10 & 20 \\
\hline Paciente $\mathrm{H}$ & 89 & 88 & 88 & 21 & 50 \\
\hline Paciente I & 66 & 82 & 71 & 3 & 10 \\
\hline Paciente J & 79 & 86 & 81 & 10 & 40 \\
\hline Média & 77,8 & 76,7 & 75,3 & 13,1 & 28,1 \\
\hline DP & 11,8 & 18,8 & 15,9 & 17,8 & 27,2 \\
\hline
\end{tabular}

O índice OP (M: 77,8; DP: 17,29) apresentou-se na faixa limítrofe $(\mathrm{Ql}=70$ a 79), o que indica dificuldade para fazer organizações lógicas, análise e síntese de material não verbal, raciocínio fluido, atenção para detalhes e integração visuomotora.

Os índices de CV (M: 81,5; DP: 11,54) e VP (M: 84; DP: 16,85) se classificaram dentro da faixa média inferior $(\mathrm{Q} I=80$ a 89), o que indica certa dificuldade em atividades verbais que avaliam o conhecimento adquirido e certa dificuldade em atividades não verbais que exigem velocidade psicomotora e capacidade de organização e de desenvolvimento de estratégias.

A figura 1 resume os resultados encontrados no subteste da WISC-III.

\section{DISCUSSÃO}

O presente estudo avaliou o funcionamento intelectual de crianças/adolescentes que sofreram exposição pré-natal ao álcool.
Analisando os escores individuais da WISC-III, pode-se perceber um continuum, já descrito na literatura², com variação de escores que configuram desde um desempenho médio até dentro da faixa de deficiência intelectual. Entretanto, nenhum indivíduo apresentou escores acima da média. Da mesma forma, uma minoria se enquadra na faixa de deficiência intelectual.

Em relação ao teste Matrizes Progressivas de Raven, o desempenho apresentado como preservado deve ser avaliado com cautela. De forma semelhante, Kodituwakku et al..$^{19}$ também encontraram desempenho intelectual médio em amostra com SAF, mas, quando comparado ao grupo controle, esse escore mostrou-se significativamente inferior. Então, para afirmar que o desempenho está realmente preservado na população avaliada, seria necessário compará-la com um grupo controle.

Em relação aos subtestes da WISC-III, o resultado limítrofe (M: 7,1; DP: 9,8) em Aritmética indica dificuldade em manejo dos números e de resolução de problemas 
aritméticos, dificuldade essa já relatada pela literatura ${ }^{20,21}$. Considerando que a maior parte da amostra investigada apresenta histórico de dificuldades acadêmicas, deve-se ponderar se fatores educacionais tiveram interferência nesse resultado.

Além disso, sujeitos que exibem um desempenho abaixo do esperado no subteste Arranjo de Figuras podem ter prejuízos secundários como o entendimento de situações sociais que envolvam habilidades não verbais, assim como em controlar os impulsos antecipando as consequências. Dificuldades em habilidades sociais, no processamento de informações sociais e de emoções faciais, já tinham sido detectadas em outros estudos 22,23 . Ações do cotidiano e do universo escolar podem sofrer o impacto de déficits dessa natureza, como organizar jogos de sequência lógica e entender e responder adequadamente a um contexto social.

Em relação aos índices gerais da WISC-III, o desempenho rebaixado em OP pode estar relacionado a déficits de processamento visual e de integração visuomotora. A dificuldade em habilidade visuomotora em indivíduos expostos ao álcool no pré-natal foi relacionada a anomalias microestruturais na substância branca em regiões temporais laterais e proximidades do esplênio lateral do corpo caloso ${ }^{24}$.

Em relação aos índices gerais da WISC-III, o desempenho rebaixado em OP pode estar relacionado a déficits de processamento visual e de integração visuomotora.

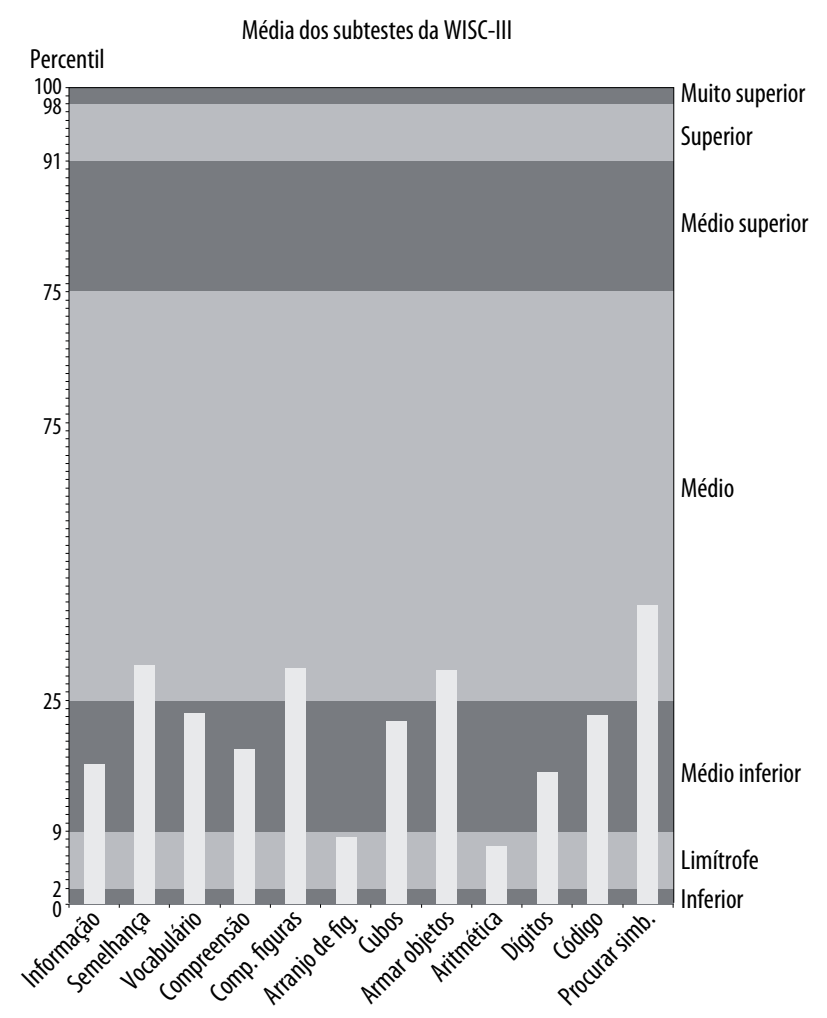

Figura 1. Média dos subtestes da WISC-III.
A dificuldade de lidar com a informação não verbal influencia tanto na forma como a pessoa lida com informações visuoespaciais (por exemplo: ler mapas ou compreender relações geométricas), como na forma como ela percebe e processa as situações sociais.

Um desempenho prejudicado nas tarefas de domínio verbal que compreendem o Índice de RD - subtestes Aritmética e Dígitos - indica que a pessoa pode apresentar dificuldades relacionadas ao processamento sequencial, atenção, memória imediata e operacional, dificuldades essas já descritas na literatura ${ }^{25-28}$.

O perfil de dificuldades que encontramos no grupo avaliado pode impactar no âmbito escolar. Assim, as crianças poderiam apresentar dificuldades na compreensão e na aprendizagem de conteúdos que dependam das habilidades matemáticas, visuoperceptivas e visuoconstrutivas.

Da mesma forma, esse perfil pode prejudicar as habilidades sociais, provocando dificuldades para compreender a linguagem não verbal, perceber detalhes e se guiar pelo todo, realizando uma leitura errônea das situações sociais. Isso pode gerar consequências inerentes à dificuldade de adaptação social: risco de bullying e tendência a isolamento social, caracterizadas como déficits secundários da TEAF.

\section{CONCLUSÕES}

De forma geral, o desempenho intelectual desta série de casos se apresentou abaixo do esperado para a população normal da mesma faixa etária, como descrito na literatura ${ }^{8-10}$. Os resultados apontam para uma dificuldade em relação à atenção e ao manejo de números e processamento visual de situações complexas.

Esta amostra apresentou maior facilidade em atividades verbais que avaliam o conhecimento adquirido e em atividades não verbais que exigem velocidade psicomotora.

Como limitação deste estudo, vale ressaltar que outros fatores, como a medicação utilizada pela criança, podem ter influenciado nos resultados apresentados. Para o estabelecimento da associação entre os efeitos do álcool e consequências neurocognitivas, seria interessante o aumento da amostra, assim como sua comparação com grupo controle.

Com os resultados apresentados, levanta-se a hipótese de que essa população pode ter sua aprendizagem escolar prejudicada, assim como as habilidades sociais, áreas que comprometem o funcionamento em todas as idades. Tornam-se necessários estudos que envolvam intervenções focadas nessas dificuldades para poder estimar o potencial de recuperação dessa população. Nosso estudo indica ainda a necessidade de incorporação de estratégias de ações para prevenção, que incluem a capacitação dos profissionais de saúde. 


\section{CONTRIBUIÇÕES INDIVIDUAIS}

Vanessa Karam de Lima Ferreira - Participou do desenho do estudo, da análise dos dados e da escrita de todo o artigo e aprovou a versão final a ser publicada.

Gisele Viegas Dias Ferreira - Participou do desenho do estudo, da análise dos dados, da escrita de todo o artigo e aprovou a versão final a ser publicada.

José Mauro Bras de Lima - Participou do desenho do estudo, revisou criticamente o conteúdo intelectual e aprovou a versão final.

Marcelo Santos Cruz - Participou do desenho do estudo, da análise dos dados e da escrita do artigo, revisou criticamente o conteúdo intelectual e aprovou a versão final.

\section{CONFLITO DE INTERESSES}

Não há conflitos de interesse a serem declarados.

\section{REFERÊNCIAS}

1. Lima JMB, Melo HVA, Netto AC. Síndrome alcoólica fetal (SAF): entidade neurológica comum, porém pouco conhecida. Rev Bras Neurol. 2006;42(3):33-40.

2. O'Leary CM. Fetal alcohol syndrome: diagnosis, epidemiology, and developmental outcomes. J Paediatr Child Health. 2004;40:2-7.

3. Streissguth AP. Fetal alcohol syndrome: a guide for families and communities. Baltimore: Paul H. Brookes Publishing Company; 1997.

4. Astley SJ. Diagnostic Guide for Fetal Alcohol Spectrum Disorders: The 4-Digit Diagnostic Code. 3rd ed. Seattle: Washington; 2004.

5. WHO. Fetal alcohol syndrome: dashed hopes, damaged lives. Bull World Health Organ. 2011;89(6):398-9.

6. Grinfeld H. Consumo nocivo de álcool durante a gravidez. In: Andrade AG, Anthony JC, Silveira CM (Org.). Álcool e suas consequências: uma abordagem multiconceitual. Barueri SP: Minha Editora; 2009. p. 179-99.

7. Lupton C, Burd L, Harwood R. Cost of fetal alcohol spectrum disorders. Am J Med Genet C Semin Med Genet. 2004;127C(1):42-50

8. Kodituwakku PW. Defining the behavioral phenotype in children with fetal alcohol spectrum disorders: a review. Neurosci Biobehav Rev. 2007;31:192-201.

9. Kaemingk KL, Mulvaney S, Halverson PT. Learning following prenatal alcohol exposure: performance on verbal and visual multitrial tasks. Arch Clin Neuropsychol. 2003;18:33-47.

10. Korkman M, Kettunen S, Autti-Rämö I. Neurocognitive impairment in early adolescence following prenatal alcohol exposure of varying duration. Child Neuropsychol. 2003;9(2):117-28.
11. Monnot M, Nixon S, Lovallo W, Ross E. Altered emotional perception in alcoholics: deficits in affective prosody comprehension. Alcohol Clin Exp Res. 2001;25(3):362-9.

12. Narberhaus A, Segarra D, Giménez $M$, Caldú X, Junqué C, Bargalló N, et al. Differential cerebral and neuropsychological consequences in dizygotic twins with prenatal alcohol exposure. Alcohol Alcohol. 2004;39(4):321-4.

13. Carlini EA, Galduróz JC, Noto AR, Carlini CM, Oliveira LG, Nappo SA, et al. Il levantamento domiciliar sobre 0 uso de drogas psicotrópicas no Brasil: estudo envolvendo as 108 maiores cidades do país - 2005. São Paulo: Páginas \& Letras; 2007.

14. Rodrigues SHP, Corradini HB. Duas síndromes fetais importantes - II. Síndrome fetal alcoólica. Pediat (S Paul0). 1981;3:309-18.

15. Stratton K, Howe C, Battaglia F. Fetal alcohol syndrome: diagnosis, epidemiology, prevention, and treatment. Washington: Institute of Medicine, National Academy Press; 1996.

16. Hoyme HE, May PA, Kalberg WO, Kodiwakku P, Gossage JP, Trujillo PM, et al. A practical approach to diagnosis of fetal alcohol spectrum disorders: clarification of the 1996 Institute of Medicine criteria. Pediatrics. 2005;115(1):39-47.

17. Figueiredo VLM. Uma adaptação brasileira do teste de inteligência WISC-III [tese]. Universidade de Brasília; 2001.

18. Raven JC. Manual de Teste Matrizes Progressivas. Rio de Janeiro: CEPA; 2002.

19. Kodituwakku P, Coriale G, Fiorentino D, Aragón AS, Kalberg W0, Buckley D, et al. Neurobehavioral characteristics of children with fetal alcohol spectrum disorders in communities from Italy: preliminary results. Alcohol Clin Exp Res. 2006;30(9):1551-61.

20. Lebel C, Rasmussen C, Wyper K, Andrew G, Beaulieu C. Brain microstructure is related to math ability in children with fetal alcohol spectrum disorder. Alcohol Clin Exp Res. 2010;34(2):354-63.

21. Burden MJ, Jacobson SW, Jacobson JL. Relation of prenatal alcohol exposure to cognitive processing speed and efficiency in childhood. Alcohol Clin Exp Res. 2005;29(8):1473-83.

22. Greenbaum RL, Stevens AS, Nash K, Koren G, Rovet J. Social cognitive and emotion processing abilities of children with fetal alcohol spectrum disorders: a comparison with attention deficit hyperactivity disorder. Alcohol Clin Exp Res. 2009;33(10):1656-70.

23. McGee CL, Bjorkquist AO, Price JM, Mattson SN, Riley EP. Social information processing skills in children with histories of heavy prenatal alcohol exposure. J Abnorm Child Psychol. 2009:37:817-30.

24. Sowell ER, Johnson A, Kan E, Lu LH, Horn JDV, Toga AW, et al. Mapping white matter integrity and neurobehavioral correlates in children with fetal alcohol spectrum disorders. J Neurosci. 2008;28(6):1313-9.

25. Astley SJ, Olson HC, Kerns K, Brooks A, Aylward EH, Coggin TE, et al. Neuropsychological and behavioral outcomes from a comprehensive magnetic resonance study of children with fetal alcohol spectrum disorders. Can J Clin Pharmacol. 2009;16(1):e178-201.

26. Dalen K, Bruarøy S, Wentzel-Larsen T, Lægreid LM. Cognitive functioning in children prenatally exposed to alcohol and psychotropic drugs. Neuropediatrics. 2009;40(4):162-7.

27. Kooistra L, Crawford S, Gibbard B, Ramage B, Kalan BJ. Differentiating attention deficits in children with fetal alcohol spectrum disorder or attention-deficit-hyperactivity disorder. Dev Med Child Neurol. 2010;52(2):205-11.

28. Aragón AS, Coriale G, Fiorentino D, Kalberg WO, Buckley D, Gossage JP, et al. Neuropsychological characteristics of Italian children with fetal alcohol spectrum disorders. Alcohol Clin Exp Res. 2008;32(11):1909-19. 